\title{
Evaluate the impact of hydrodynamic pressure on hydrologic exchange fluxes and resident time for a large-scale river section over a long-term period
}

Jie Bao ${ }^{1}$, Yunxiang Chen ${ }^{1}$, Yilin Fang ${ }^{1}$, Xuehang Song ${ }^{2}$, William Perkins ${ }^{1}$, Zhuoran Duan ${ }^{1}$, Huiying Ren ${ }^{1}$, Zhangshuan Hou ${ }^{1}$, Marshall Richmond ${ }^{1}$, Xiaoliang $\mathrm{He}^{1}$, and Timothy Scheibe $^{1}$

${ }^{1}$ Pacific Northwest National Laboratory

${ }^{2} \mathrm{PNNL}$

May 28, 2021

\begin{abstract}
Quantifying hydrologic exchange fluxes (HEF) and subsurface water residence times (RT) are important for managing the water quality and ecosystem health in dynamic river corridor systems. Laboratory-scale experiments and models have shown that hydrodynamic pressure variations on the riverbed induced by dynamic river flows can strongly impact HEFs and RTs. In this study, the impacts of hydrodynamic pressure on HEFs and RT for a $30 \mathrm{~km}$ section of the Columbia River in Washington State over a three-year period were quantitatively evaluated using a coupled transient three-dimensional (3D) multi-phase surface and subsurface water flow transport approach. Based on comparisons between model simulations with and without considering hydrodynamic pressure, we found that hydrodynamic pressure increase the net HEFs by $7 \%$ of flowing into river from subsurface domain, and also leads to a reduction of the area with long RT, and increase of area with short RT.
\end{abstract}

\section{Hosted file}

v15_rev15_NoFigurev3.docx available at https://authorea.com/users/304273/articles/523923evaluate-the-impact-of-hydrodynamic-pressure-on-hydrologic-exchange-fluxes-and-residenttime-for-a-large-scale-river-section-over-a-long-term-period

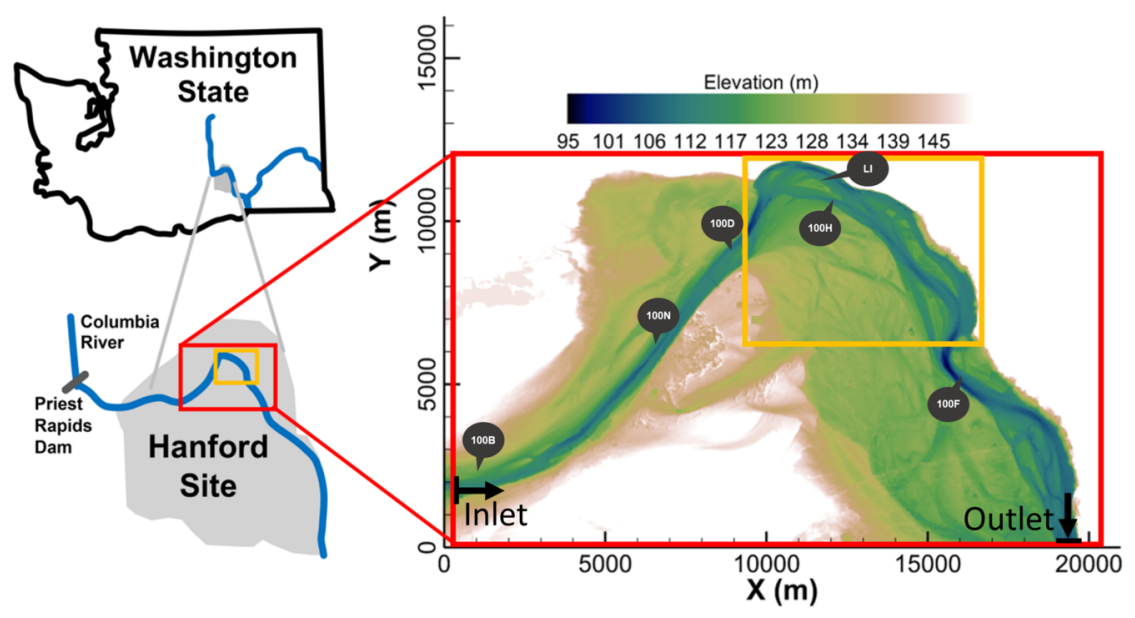




\begin{tabular}{|l|l|}
\hline Names in this study \\
Hydrodynamic/non-hydrostatic pressure \\
\hline Stagnation/total pressure
\end{tabular}

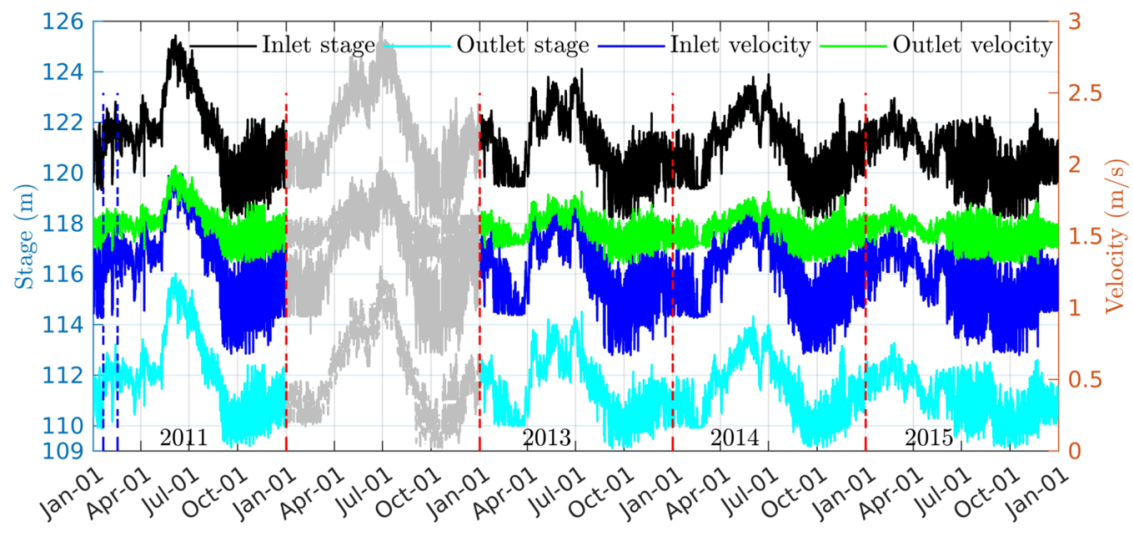

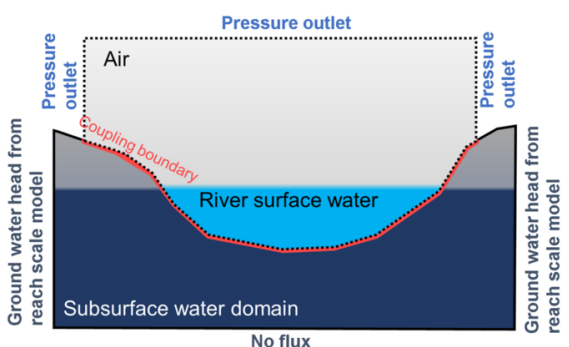

(a)

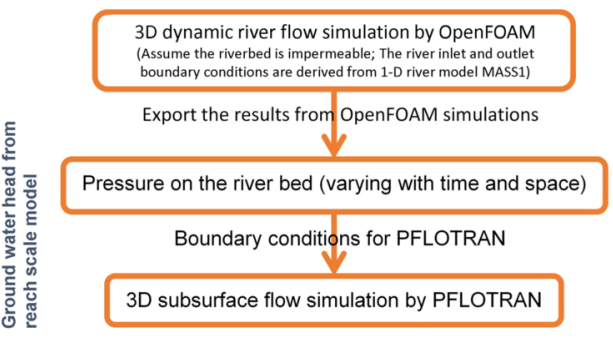

(b) 


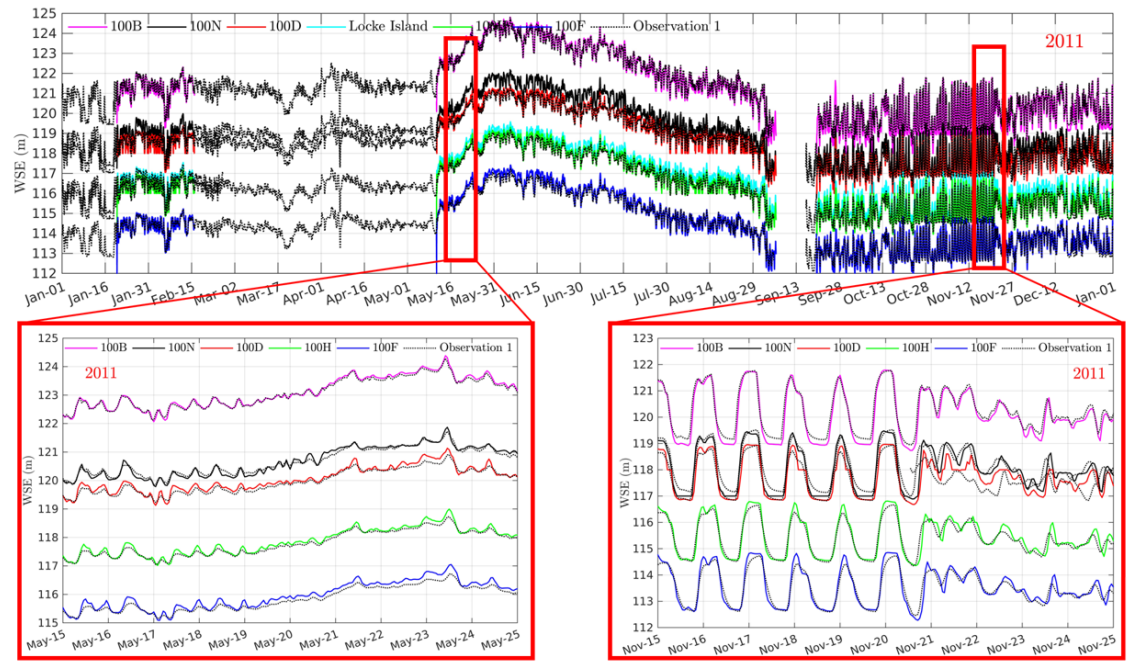

(a)

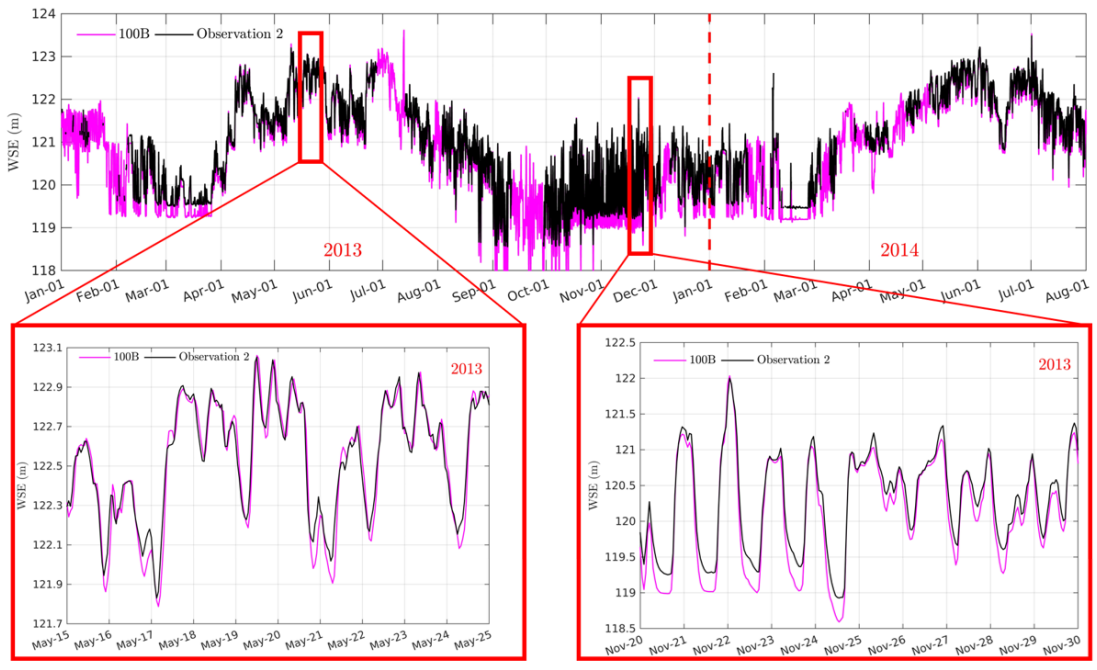

(b)

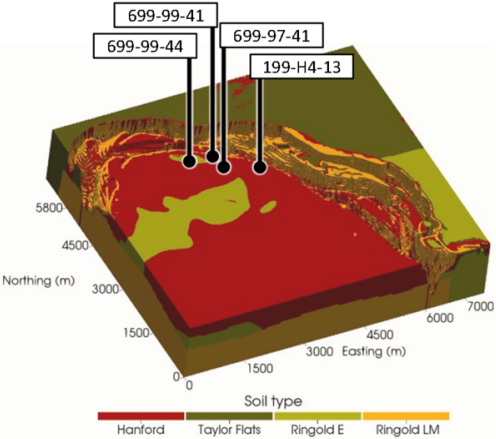

(a)

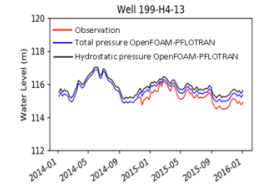

(b)

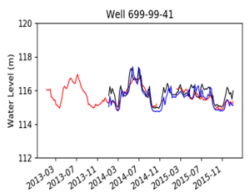

(d)

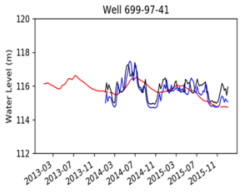

(c)

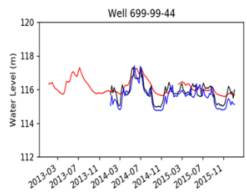

(e) 


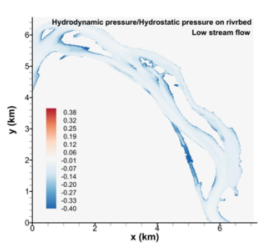

(a)

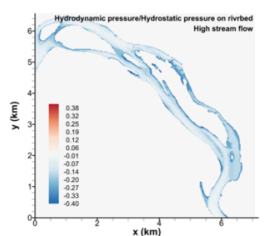

(b)

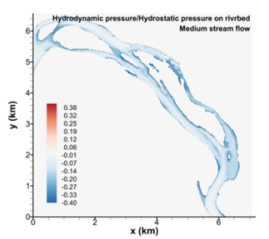

(c)

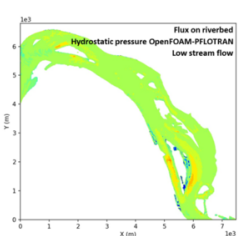

(d)

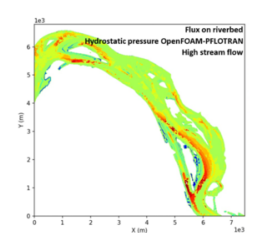

(e)

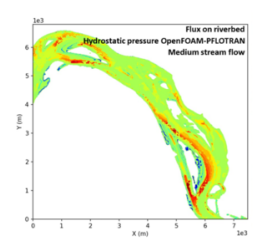

(f)

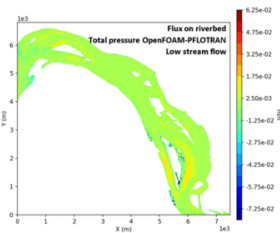

(g)

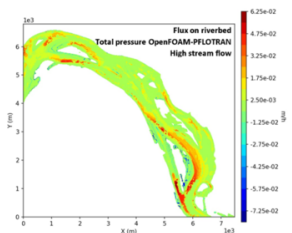

(h)

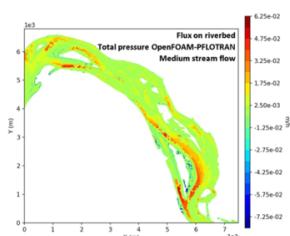

(i)

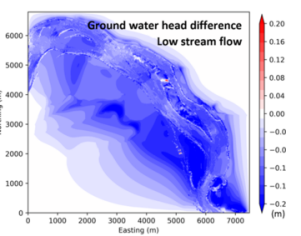

(j)

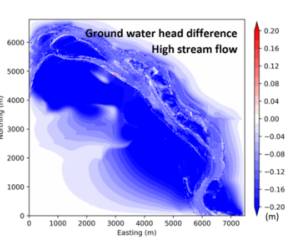

(k)

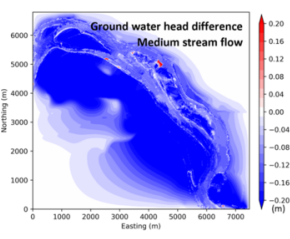

(l) 


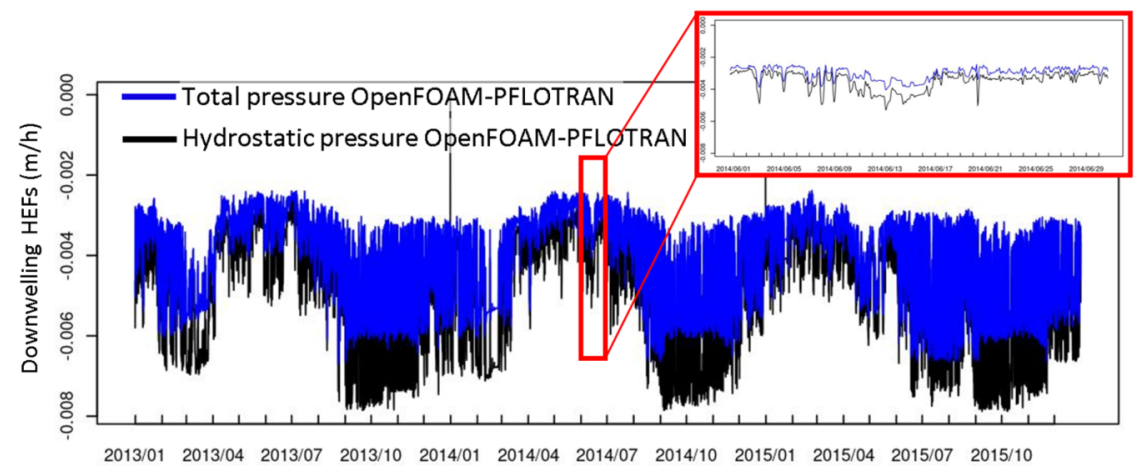

(a)

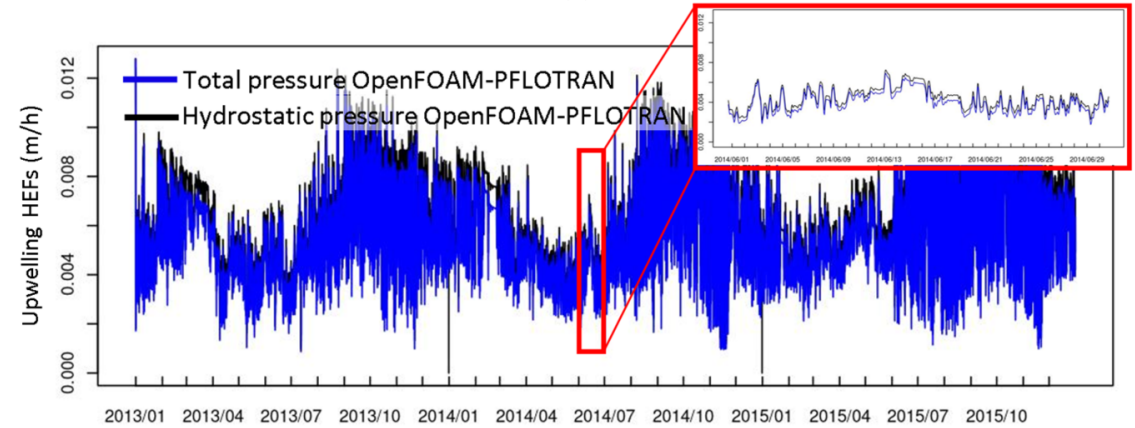

(b)

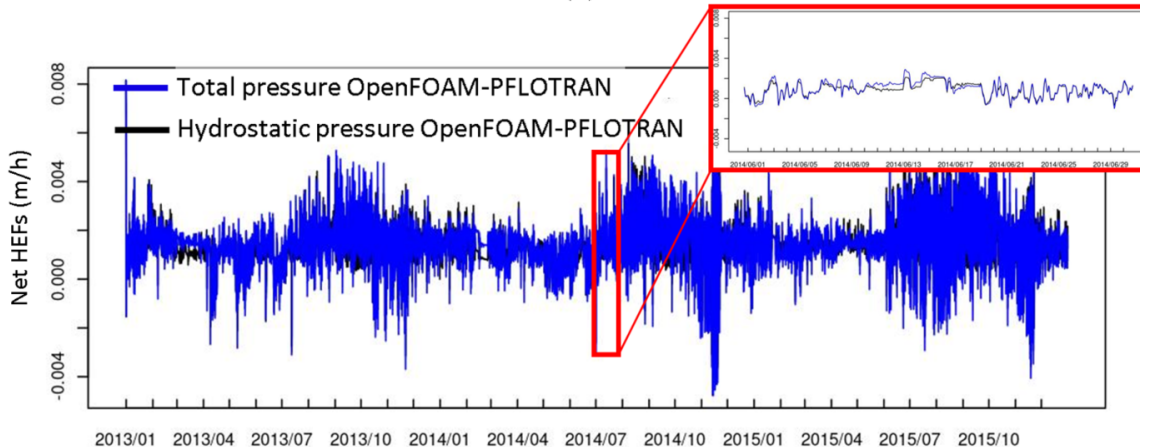

(c)
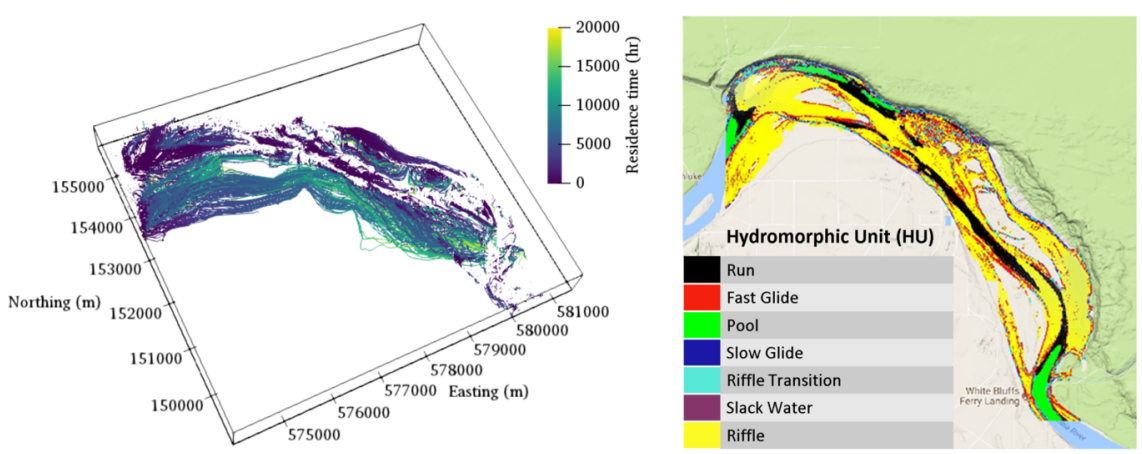

(a)

(b) 
Hydrostatic pressure OpenFOAM-PFLOTRAN (OFP static $)$

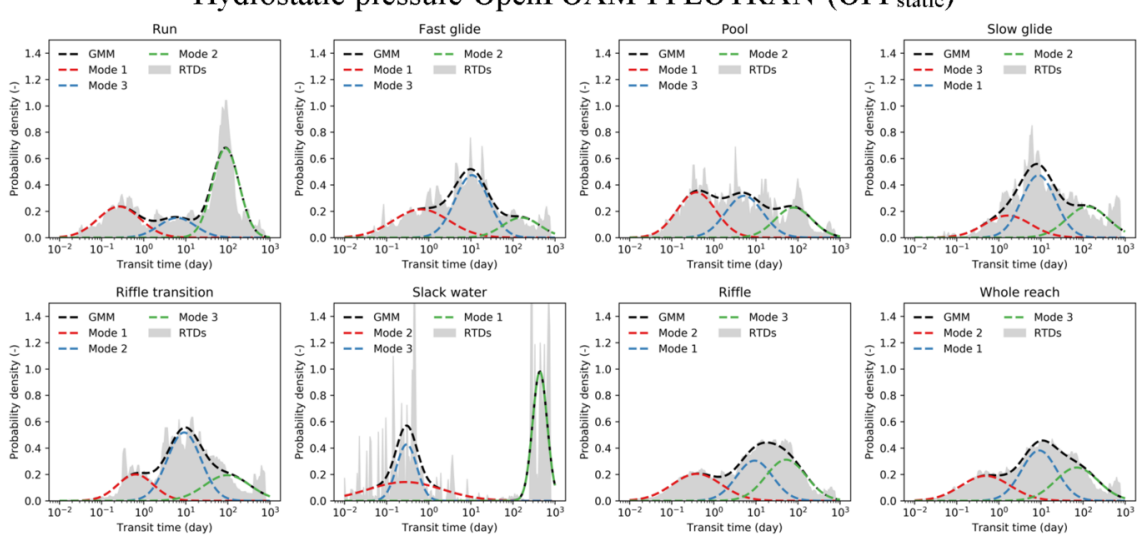

(a)

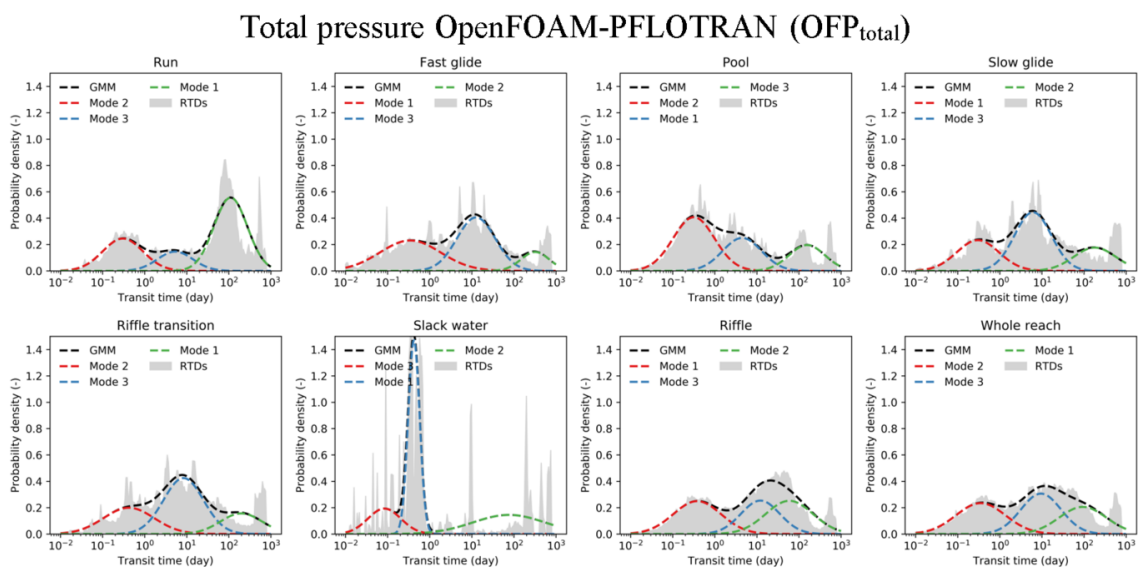

(b) 